\title{
On the asymptotic distribution of eigenvalues
}

\author{
By Eric Larsson
}

\section{Introduction}

Let $\Omega$ be the union of a finite number of open, bounded and connected subsets of $R^{n}, \Delta$ the $n$-dimensional Laplace operator and $\varrho$ a real-valued function defined in $\Omega$. Consider the eigenvalue problems

$$
\Delta f+\lambda \varrho f=0
$$

with $f$ or its normal derivative vanishing at the boundary. It has been shown by Courant ([1] p. 321) that, when $\varrho$ is a bounded Riemann integrable function and $\Omega$ satisfies a regularity condition, the asymptotic distribution of the eigenvalues is given by

$$
N(\lambda) \sim(2 \pi)^{-n} w_{n} \lambda^{n / 2} \int_{\Omega} \varrho^{n / 2}
$$

where $N(\lambda)$ stands for the number of eigenvalues smaller than $\lambda$, and $w_{n}$ is the volume of the $n$-dimensional unit sphere. The object of this note is to show that (1) holds also when $\varrho$ has a finite number of singular points $y$ in $\Omega$. More precisely, we assume that $\varrho$ is $\mathrm{O}\left(|x-y|^{-2 \beta}\right)$ in a neighbourhood of $y$, where $0<\beta<1$ when $n \geqslant 2$, and $0<\beta<1 / 2$ when $n=1$. The method adopted can also be used to treat cases when $\varrho$ becomes singular on manifolds of dimension $<n$.

We shall use the notations

\section{Preliminaries}

$$
\begin{gathered}
(f, g)_{o}=\int_{o} f \bar{g}, \quad|f|_{o}^{2}=(f, f)_{O}, \quad|f|_{o, p}=\left(\int_{o}|f|^{p}\right)^{1 / p}, \\
(\nabla f, \nabla g)_{o}=\int_{o} \nabla f \overline{\nabla g}, \quad|\nabla f|_{o}^{2}=(\nabla f, \nabla f)_{O}
\end{gathered}
$$

where $O$ is an open subset of $R^{n}$, the integrals are taken with ordinary Lebesgue measure and $\nabla f$ is the gradient of $f$, taken in the weak (distributional) sense. Whenever it is convenient we shall leave out the index $O$. 
E. LARSSON, On the asymptotic distribution of eigenvalues

Define

$$
\boldsymbol{F}_{\mathbf{1}}(O)=(f ;|\nabla f|+|f|<\infty)
$$

and let $F_{0}(O)$ be the closure in $F_{1}(O)$ of all continuously differentiable functions with compact support in $O$. The elements of $F_{0}(O)$ vanish at the boundary of $O$ at least in a weak sense. With the scalar product $(f, g)+(\nabla f, \nabla g)$ both $F_{1}(O)$ and $F_{0}(O)$ are Hilbert spaces.

An open set $O$ is said to be permitted, if it consists of a finite number of bounded and connected subsets,

the form $(f, g)$ is compact (i.e. completely continuous) in $F_{1}(O)$

and

$$
|f|_{a} \text { is majorized by a constant times }|\nabla f|+|f| \text {, }
$$

where $0<q \leqslant 2 n /(n-2)$ when $n>2$, and $0<q$ when $n=1,2$. These two properties hold if the boundary of $O$ is sufficiently smooth (see [2] p. 471 and [3] respectively). In particular, they hold when $O$ is the sum of a finite number of rectangles.

A function $\varrho \geqslant 0$ is said to be permitted in $O$ if

$$
\int_{0^{\prime}} \varrho>0 \text { for every component } O^{\prime} \text { of } O
$$

and

$$
\int_{0} \varrho^{m / 2}<\infty
$$

where $m=n$ when $n>2$, and $m$ is some number $>2$ when $n=1,2$. Let us put.

$$
(\varrho f, g)=(\varrho f, g)_{o}=\int_{o} \varrho f \bar{g} .
$$

Theorem 1. If $\varrho$ is a permitted function in a permitted set $O$, then

$$
\text { the form }(\varrho f, g) \text { is compact in } F_{1}(O)
$$

and

$$
(\varrho f, f)+|\nabla f|^{2} \sim|f|^{2}+|\nabla f|^{2} \text { in } F_{1}(O),
$$

i.e. either side is majorized by a constant times the other for all $f$ in $F_{1}(O)$.

Proof. By Hölder's inequality, (3) and (5),

$$
(\varrho f, f) \leqslant|\varrho|_{m / 2}|f|_{2 m /(m-2)}^{2} \leqslant C|\varrho|_{m / 2}\left(|f|^{2}+|\nabla f|^{2}\right),
$$

where $C$ is a constant. Hence $(\varrho f, f)$ is bounded in $F_{1}(O)$. Put $\varrho_{\lambda}(x)=\min (\lambda, \varrho(x))$. It follows from (2) that $\left(\varrho_{\lambda} f, f\right)$ is compact in $F_{1}(O)$, and (8) applied to $\varrho-\varrho_{\lambda}$ shows that $\left(\left(\varrho-\varrho_{\lambda}\right) f, f\right)$ tends to zero as $\lambda \rightarrow \infty$, uniformly on bounded sets in $F_{1}(O)$. From this $(6)$ follows.

At the same time (8) proves that, with a suitable constant $C$, 


$$
(\varrho f, f)+|\nabla f|^{2} \leqslant C\left(|f|^{2}+|\nabla f|^{2}\right) \text { in } F_{1}(O) .
$$

To prove the reverse inequality, it suffices to show that there is no sequence $\left(f_{j}\right)_{1}^{\infty}$ such that

$$
\left|\nabla f_{j}\right|^{2}+\left|f_{j}\right|^{2}=1 \text { and }\left|\nabla f_{j}\right|^{2}+\left(\varrho f_{j}, f_{j}\right) \rightarrow 0
$$

It is no restriction to assume that the sequence is weakly convergent to an element $f$ in $F_{1}(O)$. Now

$$
\left|f_{j}\right|^{2} \rightarrow|f|^{2} \text { and }\left(\varrho f_{j}, f_{j}\right) \rightarrow(\varrho f, f)
$$

since the forms are compact. In particular $(\varrho f, f)=0,\left|\nabla f_{j}\right| \rightarrow 0$ and $|f|=1$. We also have

$$
\left(\nabla f_{j}, \nabla f\right)+\left(f_{j}, f\right) \rightarrow|\nabla f|^{2}+|f|^{2}
$$

since the sequence is weakly convergent. Here

$$
\left|\left(\nabla f_{j}, \nabla f\right)\right| \leqslant\left|\nabla f_{j}\right||\nabla f|
$$

tends to zero and $\left(f_{j}, f\right)$ tends to $|f|^{2}$. Consequently

$$
|f|=1 \text { and }|\nabla f|^{2}+(\varrho f, f)=0 \text {. }
$$

But by (4), the last relation implies that $f=0$, which is a contradiction. The proof is complete.

When $\varrho$ is permitted in a permitted set $O$, we can use

$$
((f, g))=(\nabla f, \nabla g)+(\varrho f, g)
$$

as a scalar product in $F(O)=F_{0}(O)$ or $F_{1}(O)$. Then, there is a compact, selfadjoint and linear transformation $G$ defined in $F(O)$ such that

for all $f$ and $g$ in $F(O)$.

$$
(\varrho f, g)=((G f, g))
$$

From a theorem of Hilbert we have that

(a) $F(O)$ has an orthonormal basis, consisting of eigenfunctions of $G$;

(b) every eigenvalue $\mu$ of $G$ is positive and every $\mu \neq 0$ has finite multiplicity; the eigenvalues are enumerable and 0 is the only possible limit point.

If $G \varphi=\mu \varphi$, it follows that

$$
(\varrho \varphi, g)=\mu(\nabla \varphi, \nabla g)+\mu(\varrho \varphi, g) \text { when } g \in F(O),
$$

and from this by Green's formula that

with

$$
\Delta \varphi+\lambda \varrho \varphi=0
$$

$$
\lambda=(1-\mu) / \mu \text {, }
$$


where $\Delta$ is the Laplace operator, taken in the weak sense. Further, if $F(O)=$ $F_{1}(O)$, it follows that the normal derivative of $\varphi$ vanishes at the boundary and, if $F(O)=F_{0}(O)$, that $\varphi$ itself vanishes at the boundary. We shall in the following always interpret (9) in terms of the operator $G$, and $\lambda$ and $\mu$ shall be connected by $(10)$.

Our aim is to prove the asymptotic formula (1), using the well-known WeylCourant principle.

\section{Weyl-Courant's principle}

Let $\Omega$ be a permitted set and $\varrho$ a permitted function in $\Omega$, and let $\left(\Omega_{j}\right)_{j=0}^{\infty}$ be a division of $\Omega$ into permitted open subsets (their closures cover the closure of $\Omega$ ). It is clear that $\varrho$ is permitted in $\Omega_{j}$ unless (4) fails to hold in $\Omega_{j}$. Let $\left(\Omega_{j}\right)_{j=0}^{s}$ be the sets for which this does not happen, and let the function $\sigma \geqslant 0$ satisfy (5) in $\Omega$. Put

$$
H=\sum_{j=0}^{s} \oplus \boldsymbol{F}\left(\Omega_{j}\right)
$$

where $F\left(\Omega_{j}\right)=F_{0}\left(\Omega_{j}\right)$ or $F_{1}\left(\Omega_{j}\right)$, and introduce the notations

$$
\begin{aligned}
& (f, g)=\sum_{j=0}^{s}(f, g)_{\Omega_{j}}, \quad(\nabla f, \nabla g)=\sum_{j=0}^{s}(\nabla f, \nabla g)_{\Omega_{j}}, \\
& (\varrho f, g)=\sum_{j=0}^{s}(\varrho f, g)_{\Omega_{j},}, \quad(\sigma f, g)=\sum_{j=0}^{s}(\sigma f, g)_{\Omega_{j} .}
\end{aligned}
$$

As a scalar product in the Hilbert space $H$ we use

$$
((f, g))=(\nabla f, \nabla g)+(\varrho f, g)+(\sigma f, g)
$$

It is clear that $(\varrho f, f) \leqslant((f, f))$ is compact in $H$, and hence

$$
(\varrho f, g)=((G f, g)), \quad(f, G f, g \in H)
$$

defines a compact, self-adjoint and linear transformation $G$ from $H$ to $H$ such that $1>G>0$. An eigenfunction $\varphi$ of $G$ with the eigenvalue

$$
\mu=(1+\lambda)^{-1}
$$

satisfies

$$
\Delta \varphi+(\lambda \varrho-\sigma) \varphi=0
$$

in every $\Omega_{j}$, and its normal derivative vanishes at the boundary if $F\left(\Omega_{j}\right)=F_{1}\left(\Omega_{j}\right)$. Otherwise $\varphi \in F_{0}\left(\Omega_{j}\right)$ vanishes itself at the boundary. If $t=0$ except in one $\Omega_{j}$, 
$G f$ has the same property. Hence $G$ is the direct sum of its restrictions $G_{\text {, to }}$ $\Omega_{j}, 0 \leqslant j \leqslant s$. By the spectral theorem, $F\left(\Omega_{j}\right)$ has an orthonormal basis consisting of eigenfunctions of $G_{j}, 0 \leqslant j \leqslant s$, and since these are also eigenfunctions of $G$ and constitute an orthonormal basis of $H$, we have, provided every eigenvalue is counted with its multiplicity.

Theorem 2. The eigenvalues of $G$ are the union of the eigenvalues of the $G_{j}$.

Now let $\left(\varphi_{i}\right)_{1}^{\infty}$ be a complete orthonormal set of eigenfunctions of $G$ and $\left(\mu_{i}\right)_{1}^{\infty}$ the corresponding eigenvalues, ordered so that $\mu_{1} \geqslant \mu_{2} \geqslant \ldots$. Then, if

we have $\lambda_{1} \leqslant \lambda_{2} \leqslant \ldots$. Let

$$
\mu_{j}=\left(1+\lambda_{j}\right)^{-1}
$$

$$
N(\lambda)=N(\lambda, \varrho, \sigma, H)=\sum_{\lambda_{j}<\lambda} 1
$$

be the number of eigenvalues below $\lambda$. We have

Theorem 3. $N(\lambda, \varrho, \sigma, H)$ is a non-decreasing function of $\varrho$ and $H$ and $a$ nonincreasing function of $\sigma$.

Proof. It suffices to prove that $\lambda_{j}(\varrho, \sigma, H)$ has the reverse properties. The minimum-maximum principle gives

$$
\mu_{j}=\mu_{j}(\varrho, \sigma, H)=\inf _{L} \sup _{f \in L}(\varrho f, f) /((f, f)),
$$

where $L$ runs through all subspaces of $H$ of codimension $<j$. Since

$$
\mu_{j}=\left(1+\lambda_{j}\right)^{-1}
$$

we get

$$
\lambda_{j}=\lambda_{j}(\varrho, \sigma, H)=\sup _{L} \inf _{f \in L}\left(|\nabla f|^{2}+(\sigma f, f)\right) /(\varrho f, f)
$$

Hence, it is clear that $\lambda_{j}$ is a non-increasing function of $\varrho$ and a non-decreasing function of $\sigma$. Next, let $H^{\prime} \supset H$ be of the same type as $H$. Since $\operatorname{cod} L<j$ in $H$, there is a subspace $M^{\prime} \subset H^{\prime}$ of dimension $<j$ such that $f \in H$ and $f \perp M^{\prime}$ implies $f \in L$. Hence,

$$
\lambda_{j}(\varrho, \sigma, H)=\sup _{M^{\prime}} \alpha\left(M^{\prime}\right)
$$

where $\alpha\left(M^{\prime}\right)=\inf \left(|\nabla f|^{2}+(\sigma f, f)\right) /(\varrho f, f)$ when $f \in H$ and $f \perp M^{\prime}$.

Replacing $H$ by $H^{\prime}$, we get a new function

$$
\alpha^{\prime}\left(M^{\prime}\right) \leqslant \alpha\left(M^{\prime}\right) .
$$

Since

$$
\sup _{M^{\prime}} \alpha^{\prime}\left(M^{\prime}\right)=\lambda_{j}\left(\varrho, \sigma, H^{\prime}\right)
$$


E. LARSSON, On the asymptotic distribution of eigenvalues

This completes the proof.

$$
\lambda_{j}(\varrho, \sigma, H) \geqslant \lambda_{j}\left(\varrho, \sigma, H^{\prime}\right)
$$

We conclude this section by proving two lemmas, which will be used later.

Lemma 1. If $\varrho \geqslant 1$ is a permitted function in a permitted set $O$, then

$$
N\left(\lambda, \varrho, 0, F_{1}(O)\right) \leqslant N\left((1+v) \lambda+v, \varrho, \sigma, F_{1}(O)\right),
$$

where

$$
v=C|\sigma|_{m / 2}
$$

with $C$ depending only on $m$ and $O$.

Proof. By Hölder's inequality, (3) and (5),

Hence, since $\varrho \geqslant \mathrm{I}$ in $O$,

$$
(\sigma f, f) \leqslant C|\sigma|_{m / 2}\left(|\nabla f|^{2}+|f|^{2}\right)
$$

$$
\left(\left|\nabla f^{2}\right|+(\sigma f, f)\right) /(\varrho f, f) \leqslant(1+v)|\nabla f|^{2} /(\varrho f, f)+v .
$$

Consequently, with $L$ running through all subspaces of $F_{1}(O)$ of codimension $<j$,

$$
\begin{aligned}
& \lambda,\left(\varrho, \sigma, F_{1}(O)\right)=\sup _{L} \inf _{f \in L}\left(|\nabla f|^{2}+(\sigma f, f)\right) /(\varrho f, f) \\
& \quad \leqslant \sup _{L} \inf _{f \in L}\left((1+v)|\nabla f|^{2} /(\varrho f, f)+v\right)=(1+v) \lambda_{j}\left(\varrho, 0, F_{1}(O)\right)+v .
\end{aligned}
$$

Thus, $\lambda_{j}\left(\varrho, 0, F_{1}(O)\right)<\lambda$ implies $\lambda_{j}\left(\varrho, \sigma, F_{1}(O)\right)<(1+v) \lambda+v$ and the lemma follows.

Lemma 2. If $G \varphi=\mu \varphi$ and $\mu=(1+\lambda)^{1}$, then the support of $\varphi$ cannot be contained in the set where $\lambda \varrho-\sigma<0$.

Proof. $\quad G \varphi=\mu \varphi$ gives $(\varrho \varphi, \varphi)=\mu((\varphi, \varphi))$, i.e.

$$
|\nabla \varphi|^{2}=((\lambda \varrho-\sigma) \varphi, \varphi)
$$

and hence we have the lemma.

\section{The asymptotic formula}

The case, where $\Omega$ is a $n$-dimensional rectangle, $\varrho$ a constant and $H=F_{0}(\Omega)$ or $F_{1}(\Omega)$, is classical. Since $(\nabla f, \nabla g)$ is invariant under translations and orthogonal transformations, we can assume that

$$
\Omega=\left(x ; 0<x_{k}<a_{k}, 1 \leqslant k \leqslant n\right) .
$$

Then the eigenfunctions are

$$
\prod_{j=1}^{n} \sin \pi l_{j} x_{,} a_{j}{ }^{-1}, \quad \text { if } \quad H=F_{0}(\Omega),
$$


and

$$
\prod_{j=1}^{n} \cos \pi l_{j} x_{j} a_{j}^{-1}, \quad \text { if } \quad H=F_{1}(\Omega),
$$

where $l_{j}=1,2,3, \ldots$ in the first and $0,1,2,3, \ldots$ in the second case. Thus, the eigenvalues are

$$
\pi^{2} \varrho^{-1} \sum_{j=1}^{n}\left(l_{j} / a_{j}\right)^{2}
$$

which gives

$$
N(\lambda, \varrho, 0, H)=(2 \pi)^{-n} w_{n} \int_{\Omega}(\lambda \varrho)^{n / 2}+O\left(\lambda^{(n-1) / 2}\right),
$$

where $w_{n}$ is the volume of the $n$-dimensional unit sphere, $\left(w_{1}=2\right)$. This estimate will be used later. We shall also need

Lemma 3. If $a_{i} \leqslant a$ and $a_{l} \leqslant b$ when $l \neq i$, then

$$
N\left(\lambda, \varrho, 0, F_{1}(\Omega)\right) \leqslant 2^{n-1}\left(1+b^{n-1}(\lambda \varrho)^{(n-1) / 2}\right)\left(1+a(\lambda \varrho)^{\frac{1}{2}}\right) .
$$

Proof. The number of non-negative integral solutions of

$$
\sum_{j=1}^{n}\left(l_{j} / a_{j}\right)^{2}<\pi^{-2} \varrho \lambda
$$

is majorized by

$$
\prod_{j=1}^{n}\left(1+\pi^{-1} a_{j}(\varrho \lambda)^{\frac{1}{2}}\right)
$$

so that the lemma follows.

Now, to simplify the notations, write

$$
\begin{aligned}
& \bar{N}(\varrho, \sigma, H)=\lim \sup \lambda^{-n / 2} N(\lambda, \varrho, \sigma, H), \quad(\lambda \rightarrow \infty), \\
& \underline{N}(\varrho, \sigma, H)=\lim \inf \lambda^{-n / 2} N(\lambda, \varrho, \sigma, H), \quad(\lambda \rightarrow \infty)
\end{aligned}
$$

and $N(\varrho, \sigma, H)=\bar{N}=\underline{N}$ when the limits are equal. Also, put

$$
M(\varrho, \Omega)=(2 \pi)^{-n} w_{n} \int_{\Omega} \varrho^{n / 2} .
$$

When

$$
\Omega=\left(\Omega_{j}\right)_{j=0}^{p}
$$

is a sum of rectangles, and $\varrho$ and $\bar{\varrho}$ are constants in these rectangles such that $\varrho \leqslant \varrho$ and $0<\bar{\varrho} \geqslant \varrho$, Theorem 2 and Theorem 3 give

$$
\Sigma^{\prime} N\left(\lambda, \underline{\varrho}, 0, F_{0}\left(\Omega_{j}\right)\right) \leqslant N\left(\lambda, \varrho, 0, F_{0}(\Omega)\right) \leqslant N\left(\lambda, \varrho, 0, F_{1}(\Omega)\right) \leqslant \sum N\left(\lambda, \bar{\varrho}, 0, F_{1}\left(\Omega_{j}\right)\right),
$$

where $\Sigma^{\prime}$ denotes that we only sum over such $j$ that $\varrho>0$ in $\Omega_{j}$. Hence,

$$
M(\varrho, \Omega) \leqslant \underline{N}\left(\varrho, 0, F_{0}(\Omega)\right) \leqslant \bar{N}\left(\varrho, 0, F_{1}(\Omega)\right) \leqslant M(\bar{\varrho}, \Omega) .
$$




\section{E. LARSSON, On the asymptotic distribution of eigenvalues}

If $\varrho$ is bounded and Jordan measurable, the first and the last term can be made arbitrarily close by choosing a fine subdivision of $\Omega$, and hence

$$
N(\varrho, 0, F)=M(\varrho, \Omega) \text {, }
$$

where

$$
F=F_{0}(\Omega) \text { or } F_{1}(\Omega)
$$

This is the asymptotic formula in this very regular case. We shall see that the same formula holds also when $\varrho$ has moderate singularities, more precisely, if

(a) $\Omega$ is a finite sum of rectangles,

(b) $\varrho \geqslant 0, \int_{\Omega} \varrho>0$ and $\varrho$ is bounded except for a finite number of singular points $y$ in $\Omega$, where

$$
\varrho(x)=O\left(|x-y|^{-2 \beta}\right)
$$

and

with $0<\beta<1$ when $n \geqslant 2$, and $0<\beta<\frac{1}{2}$ when $n=1$,

(c) $\varrho$ is Jordan measurable.

For convenience, the norm $|x|$ is defined as $\max _{j}\left|x_{j}\right|$. We have made the first assumption, since we are interested only in the singularities of the function $\varrho$ and not in the complications that arise from the boundary. For generalization to more general regions we refer to [1]. The third condition implies that $\varrho^{n / 2}$ is Riemann integrable.

Now, let $\Omega_{0}$ be such a sum of rectangular neighbourhoods of the $y$ that $\Omega-\Omega_{0}$ is also a sum of rectangles. Then we have by Theorem 2 and Theorem 3

$$
\begin{aligned}
N\left(\lambda, \varrho, 0, F_{0}\left(\Omega-\Omega_{0}\right)\right) \leqslant N\left(\lambda, \varrho, 0, F_{0}(\Omega)\right) & \leqslant N\left(\lambda, \varrho, 0, F_{1}(\Omega)\right) \leqslant N\left(\lambda, \varrho, 0, F_{1}\left(\Omega_{0}\right)\right) \\
& +N\left(\lambda, \varrho, 0, F_{1}\left(\Omega-\Omega_{0}\right)\right)
\end{aligned}
$$

and hence

$$
M\left(\varrho, \Omega-\Omega_{0}\right) \leqslant \underline{N}\left(\varrho, 0, F_{0}(\Omega)\right) \leqslant \vec{N}\left(\varrho, 0, F_{1}(\Omega) \leqslant \bar{N}\left(\varrho, 0, F_{1}\left(\Omega_{0}\right)\right)+M\left(\varrho, \Omega-\Omega_{0}\right) .\right.
$$

Here we see that in order that

$$
N(\varrho, 0, F(\Omega))=M(\varrho, \Omega)
$$

with $F=F_{0}$ or $F_{1}$, it is sufficient to prove that

$$
\bar{N}\left(\varrho, 0, F_{1}\left(\Omega_{0}\right)\right) \rightarrow 0
$$

as the diameter of $\Omega_{0}$ tends to zero. Hence, if

we have, putting

$$
D_{t} \text { stands for the cube }|x|<t
$$

that it suffices to show that

$$
\xi(x)=|x|^{-2 \beta}
$$




$$
\mathbb{N}\left(\xi, 0, F_{1}\left(D_{\tau}\right)\right) \rightarrow 0 \quad \text { when } r \rightarrow 0 .
$$

Consider

$$
N(\lambda)=N\left(\lambda, \xi, 0, F_{1}(D)\right),
$$

where $D=D_{r}$. When $\xi \geqslant 1$ in $D$ and $\sigma \geqslant 0$ satisfies (5), we have according to Lemma 1

$$
N\left(\lambda, \xi, 0, F_{1}(D)\right) \leqslant N\left((1+v) \lambda+v, \xi, \sigma, F_{1}(D)\right),
$$

where

$$
v=C|\sigma|_{m i 2}
$$

with $C$ depending on $m$ and $D$ and hence on $r$.

We put

$$
\sigma(x)=|x|^{-2(\beta+\varepsilon)}
$$

$$
\left(\beta<\beta+\varepsilon<1 \text { when } n \geqslant 2, \quad \beta<\beta+\varepsilon<\frac{1}{2} \text { when } n=1\right)
$$

inside a cube $D^{\prime}$ with its centre at the origin and

$$
\sigma(x)=0
$$

outside, and choose $D^{\prime}$ so small that $v \leqslant 1$. Then

$$
N\left(\lambda, \xi, 0, F_{1}(D)\right) \leqslant N\left(\lambda^{\prime}, \xi, \sigma, F_{1}(D)\right),
$$

where $\lambda^{\prime}=2 \lambda+1$. Consider $D_{s} \subset D$. By Theorem 2 and Theorem 3

$$
N\left(\lambda^{\prime}, \xi, \sigma, F_{1}(D)\right) \leqslant N\left(\lambda^{\prime}, \xi, 0, F_{1}\left(D-D_{s}\right)\right)+N\left(\lambda^{\prime}, \xi, \sigma, F_{1}\left(D_{s}\right)\right) .
$$

Now determine $s=s(\lambda)$ so that $D_{s} \subset D^{\prime}$ and

$$
\lambda^{\prime} \xi-\sigma<0 \text { in } D_{s} .
$$

This is possible, if e.g. $\quad \lambda^{\prime}=2 \lambda+1=s^{2}$,

and $\lambda$ is sufficiently large.

Then, by Lemma 2, the second term on the right side of (14) vanishes, and we get

$$
N(\lambda) \leqslant N\left(\lambda^{\prime}, \xi, 0, F_{1}\left(D-D_{s}\right)\right) .
$$

Now it is easy to estimate the right side.

We choose $p$ numbers such that

$$
r=r_{0}>r_{1}>\ldots r_{p}=s .
$$

A more precise choise will be made later. Put $D_{f}=D_{r_{j}}$. Then by Theorem 2 and Theorem 3,

$$
N(\lambda) \leqslant \sum_{j=0}^{p-1} N\left(\lambda^{\prime}, \xi, 0, F_{1}\left(D_{j}-D_{j+1}\right)\right) .
$$


E. IAASSON, On the asymptotic distribution of eigenvalues

Now $D_{j}-D_{j+1}$ is obviously a sum of a fixed number of rectangular regions of diameter $\leqslant r_{j}$, having one side equal to $\left(r_{j}-r_{j+1}\right)$. Further, $\xi \leqslant r_{j+1}^{-2 \beta}$ in $D_{j}-D_{j+1}$. Hence, by (15), (16), Lemma 3 and Theorem 3, there is a constant $C$ such that

and hence

$$
N(\lambda) \leqslant C \sum_{j=0}^{p-1}\left(1+r_{j}^{n-1} r_{j+1}^{-\beta(n-1)} \lambda^{(n-1) / 2}\right)\left(1+r_{j+1}^{-\beta}\left(r_{j}-r_{j+1}\right) \lambda^{\frac{1}{j}}\right)
$$

$$
\lambda^{-n / 2} N(\lambda) \leqslant C \sum_{j=0}^{D-1}\left((\lambda)^{-(n \cdot 1) / 2}+r_{j}^{n-1} r_{j+1}^{-\beta(n-1)}\right)\left((\lambda)^{-\frac{1}{2}}+r_{j+1}^{-\beta}\left(r_{j}-r_{j+1}\right)\right) .
$$

By virtue of (15) we may write

$$
\lambda^{n / 2} N(\lambda) \leqslant C \sum_{j-0}^{p-1}\left(s^{(n-1) \varepsilon}+r_{j}^{n-1} r_{j+1}^{-\beta(n-1)}\right)\left(s^{e}+r_{j+1}^{-\beta}\left(r_{j}-r_{j+1}\right)\right)
$$

provided we increase the constant.

We now choose the numbers $r_{j}$ so that

$$
2 \leqslant r_{j} / r_{j+1} \leqslant 4 \text { for all } j \text {. }
$$

It is easy to see that this is always possible if $s<r / 2$. Then we have

and hence

$$
s=r_{D} \leqslant 2^{-p} r
$$

$$
s^{s} p \rightarrow 0 \text { as } s \rightarrow 0 \text {. }
$$

Further, (17) gives, with still another $C$,

$$
\lambda^{-n / 2} N(\lambda) \leqslant C \sum_{j=0}^{p-1}\left(s^{(n-1) e}+r_{j}^{(n-1)(1-\beta)}\right)\left(s^{8}+r_{j}^{\beta}\left(r_{j}-r_{j+1}\right)\right) .
$$

Since $1-\beta>0$, we obtain the following majorant for the right side

and hence by (18)

$$
C\left(s^{(n-1) \varepsilon}+r^{(n-1)(1-\beta)}\right)\left(s^{\varepsilon} p+\int_{0}^{r} t^{-\beta} d t\right)
$$

$$
\lim \sup \lambda^{-n / 2} N(\lambda) \leqslant C r^{(n-1)(1-\beta)} \int_{0}^{r} t^{-\beta} d t==O\left(r^{n(1-\beta)}\right)
$$

which tends to zero with $r$ and the proof is finished.

Remark. Using the Weyl-Courant principle and the fact that $\xi$ is homogeneous of order $-2 \beta$, it is easy to see that

This gives

$$
\lambda_{f}\left(\xi, 0, F_{1}\left(D_{r}\right)\right)=r^{2(\beta-1)} \lambda_{j}\left(\xi, 0, F_{1}\left(D_{1}\right)\right)
$$




$$
N\left(\lambda, \xi, 0, F_{1}\left(D_{r}\right)\right)=N\left(r^{2(1-\beta)} \lambda, \xi, 0, F_{1}\left(D_{1}\right)\right) .
$$

Hence (13) is a consequence of

$$
\bar{N}\left(\xi, O, F_{1}\left(D_{1}\right)\right)<\infty,
$$

and this follows if we put $r=1$ in the proof above.

\section{RE FER E N CES}

1. Couran'T-Hutuert, Methoden der matematischen Physik, $\mathrm{I}$.

2. Courant-Hitubert, Methoden der matematischen Physik, II.

3. Deny-Lrons, Les espaces du type de Beppo Levi. Annales de l'Institut Fourier, 5. 